\title{
Lengthening a Tetrahedron
}

\author{
Richard Evan Schwartz *
}

August 11, 2014

\begin{abstract}
We give rigorous, computer assisted proofs of a number of statements about the effect on the volume of lengthening various edges of a tetrahedron. Our results give new and sharp polynomial inequalities concerning the Cayley-Menger determinant and its partial derivatives.
\end{abstract}

\section{Introduction}

This paper was inspired by a question posed by Daryl Cooper: Suppose that you lengthen all the sides of a tetrahedron by one unit. Is the result still a tetrahedron, and (if so) does the volume increase? More formally, say that a list $\left\{d_{i j} \mid i \neq j \in\{1,2,3,4\}\right\}$ is tetrahedral if there are 4 distinct points $V_{1}, V_{2}, V_{3}, V_{4} \in \boldsymbol{R}^{3}$ so that $d_{i j}=\left\|V_{i}-V_{j}\right\|$ for all $i, j$. We call the list $\left\{d_{i j}+1\right\}$ the unit lengthening of $\left\{d_{i j}\right\}$.

Theorem 1.1 The unit lengthening of a tetrahedral list is also tetrahedral. If $\Delta_{0}$ is the original tetrahedron and $\Delta_{1}$ is the new tetrahedron, then

$$
\frac{\operatorname{volume}\left(\Delta_{1}\right)}{\operatorname{volume}\left(\Delta_{0}\right)} \geq\left(1+\frac{6}{\sum_{i<j} d_{i j}}\right)^{3}
$$

The inequality is sharp, because it is an equality for all regular tetrahedra.

We also have the following general result.

Theorem 1.2 In every dimension, the unit lengtening of a simplicial list is again simplicial, and the new simplex has volume larger than the original.

* Supported by N.S.F. Research Grant DMS-1204471 
Here a simplicial list is the obvious generalization of a tetrahedral list to higher dimensions. One could say that Theorem 1.2 is new, and one could say that it has been there all along. After discussing an earlier version of this paper with Peter Doyle and Igor Rivin, they realized that the general result follows from a theorem, [WW, Corollary 4.8], attributed to Von Neumann. I'll give the argument in an appendix. It is independent from the rest of the paper.

Theorem 1.1 relies on a sharp inequality concerning the Cayley-Menger determinant and one of its directional derivatives. Let $K_{4}$ be the complete graph on 4 vertices. Say that a pseudo-tetrahedron is a non-negative labeling of the edges of $K_{4}$ so that, going around any 3-cycle of $K_{4}$, the edges satisfy the triangle inequality. Let $X$ denote the space of pseudotetrahedra. We think of $X$ as a polyhedral cone in $\boldsymbol{R}^{6}$ by considering the points $\left(d_{12}, d_{13}, d_{14}, d_{23}, d_{24}, d_{34}\right)$.

Given a pseudo-tetrahedron $D=\left\{d_{i j}\right\}$ we have the famous CayleyMenger determinant

$$
f(D)=\operatorname{det}\left[\begin{array}{ccccc}
0 & 1 & 1 & 1 & 1 \\
1 & 0 & d_{12}^{2} & d_{13}^{2} & d_{14}^{2} \\
1 & d_{21}^{2} & 0 & d_{23}^{2} & d_{24}^{2} \\
1 & d_{31}^{2} & d_{32}^{2} & 0 & d_{34}^{2} \\
1 & d_{41}^{2} & d_{42}^{2} & d_{43}^{2} & 0
\end{array}\right]
$$

When $D$ represents a tetrahedron $T_{D}$, we have the following classic result.

$$
f(D)=288 V^{2}=2^{3} \times(3 ! V)^{2}, \quad V=\operatorname{volume}\left(T_{D}\right) .
$$

See $[\boldsymbol{P}]$ for a proof, and $[\mathbf{S a}]$ for a vast survey of generalizations. We also define the directional derivative

$$
g=D_{(1,1,1,1,1,1)} f .
$$

$f$ is a homogeneous polynomial of degree 6 and $g$ is a homogeneous polynomial of degree 5. Theorem 1.1 is a quick consequence of the following result.

Theorem 1.3 Let $C$ be a constant. The function $g \sum_{i<j} d_{i j}-C f$ is nonnegative on $X$ if and only if $C \in[16,36]$. 
We will reduce Theorem 1.3 to the statement that a certain polynomial in $Z\left[X_{1}, \ldots, X_{5}\right]$ is non-negative on the unit cube $[0,1]^{5}$. We then use about an hour of exact integer calculation in Java to establish the non-negativity. $\S 3$ describes the method and $\S 4$ gives details about its implementation in this case. I call it the Method of Positive Dominance. I have no idea if it is a known technique, though I also used it in $[\mathbf{S}]$.

In $\S 5$, we will use the same methods to prove generalizations of Theorems 1.1 and 1.3 which deal with selectively lengthening some subset of the edges of a tetrahedron. Here is the framework for these results. Each pseudotetrahedron gives rise to 4 vertex sums and 3 axis sums. A vertex sum is the sum of the labels of 3 edges incident to a given vertex - e.g. $d_{12}+d_{13}+d_{14}$. An axis sum is the sum of labels of 2 opposite edges - e.g. $d_{12}+d_{34}$.
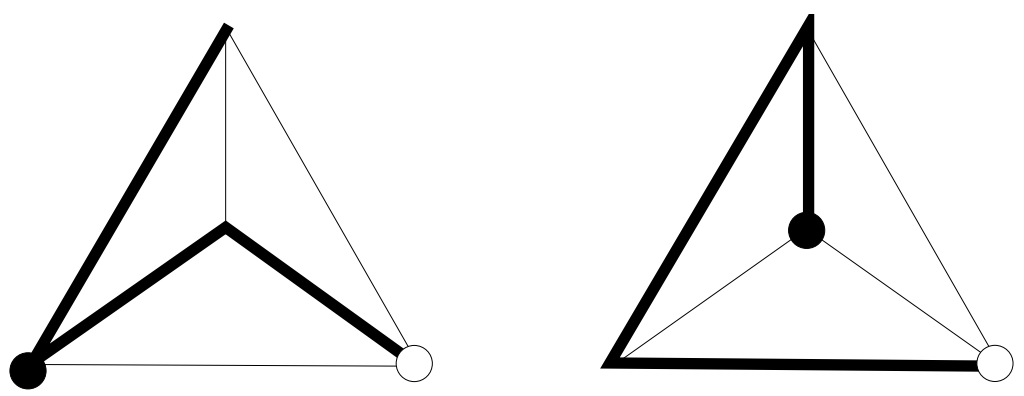

Figure 1.1: 2 of the 48 decorations of $K_{4}$.

Figure 1.1 shows 2 of the 48 possible decorations of $K_{4}$ in which we choose an embedded 3-path, a white endpoint of the path, and a black vertex of the path which is not adjacent to the white endpoint. For each such decoration $D$, we have a subset $X_{D} \subset X$ consisting of those pseudo-tetrahedra with the following properties:

- The axis sum of the opposite pair contained in $D$ is largest.

- The axis sum of the opposite pair disjoint from $D$ is smallest.

- The vertex sum at the black vertex is smallest.

- The vertex sum at the white vertex is not greater than the vertex sum at the vertex of $D$ incident to the white vertex. 
It turns out that $X_{D}$ is linearly isomorphic to an orthant in $\boldsymbol{R}^{6}$. We call $X_{D}$ a chamber. Our construction partitions $X$ into 48 chambers. We will explore this partition more thoroughly in $\S 2$.

Let $\beta \subset K_{4}$ denote a subset of edges. Call $\beta$ friendly if $K_{4}-\beta$ is not a union of edges all incident to the same vertex. Otherwise, we call $\beta$ unfriendly. Up to isometry, there are 7 friendly subsets and 3 unfriendly ones.

Theorem 1.4 Let $\beta \subset K_{4}$ denote any friendly subset. Let $g=D_{\beta} f$ denote the directional derivative of $f$ along $\beta$. There is a nonempty union $X_{\beta}$ of chambers of $X$, and constants $A_{\alpha}<B_{\beta}$, with $B_{\beta}>0$, such that the function $g \sum_{i<j} d_{i j}-C f$ is non-negative on $X_{\beta}$ if and only if $C \in\left[A_{\alpha}, B_{\beta}\right]$. Moreover, every chamber of $X-X_{\beta}$ contains a point where $f>0$ and $g<0$.

When $\beta=K_{4}$, Theorem 1.3 tells us that $X_{\beta}=X$ and $\left(A_{\beta}, B_{\beta}\right)=(16,36)$. Here is a summary of what we prove in the remaining cases.

- When $\beta$ is a single edge, $X_{\beta}$ consists of the 12 chambers $X_{D}$ such that $\beta \not \subset D$ and the black vertex of $D$ is an endpoint of $\beta$. (See Figure 5.1.) Here $\left(A_{\beta}, B_{\beta}\right)=(0,2)$.

- When $\beta$ is a pair of incident edges, $X_{\beta}$ is the set of 4 chambers $X_{D}$ such that $\beta$ is disjoint from the outer two edges of $D$ and the black dot is incident to both edges of $\beta$. (See Figure 5.2) Here $\left(A_{\beta}, B_{\beta}\right)=(0,12)$.

- When $\beta$ is a pair of opposite edges, $X_{\beta}$ is the set of 32 chambers $X_{D}$ such that $\beta \not \subset D$. (See Figure 5.3 for some pictures.) The constants satisfy $A_{\beta} \leq 0$ and $B_{\beta} \geq 4$.

- When $\beta$ is 3 edges incident to a vertex $v$, the set $X_{\beta}$ consists of the 12 chambers $X_{D}$ such that the black vertex is $v$. Here $\left(A_{\beta}, B_{\beta}\right)=(8,18)$.

- When $\beta$ is a 3-path, $X_{\beta}$ is the set of 8 chambers $X_{D}$ such that the black vertex is an interior vertex of $\beta$, and the outer edges of $D$ are disjoint from $\beta$. Here $A_{\beta} \leq-6$ and $B_{\beta} \geq 16$.

- When $\beta$ is a 4-cycle, $X_{\beta}$ consists the 16 chambers $X_{D}$ such that the outer two edges of $D$ are disjoint from $\beta$. Here $\left(A_{\beta}, B_{\beta}\right)=(0,24)$. 
We have declared 3-cycles unfriendly, but actually we can say a lot about what happens for them. When $\beta$ is a 3 -cycle, let $X_{\beta}$ denote the 36 chambers $X_{D}$ so that the black vertex lies in $\beta$.

Theorem 1.5 Let $\beta$ be a 3-cycle. Let $g=D_{\beta} f$ denote the directional derivative of $f$ along $\beta$. Then the function $g \sum_{i<j} d_{i j}-C f$ is non-negative on $X_{\beta}$ if and only if $C=8$. Moreover, every chamber of $X-X_{\beta}$ contains a point where $f>0$ and $g<0$.

Remark: Roughly speaking, the decorations defining $X_{\beta}$ try as hard as possible to have their edges disjoint from $\beta$, and their marked vertices contained in $\beta$.

Say that a lengthening of a tetrahedron along a subset of edges locally increases (respectively decreases) the volume if the volume goes up (respectively down) when we add the same sufficiently small amount to each edge in the subset. Theorems 1.4 and 1.5 have the following immediate corollary.

Corollary 1.6 Suppose that $\beta$ is either a 3-cycle or a friendly subset of $K_{4}$. For any tetrahedron in $X_{\beta}$, the lengthening along $\beta$ locally increases volume. Moreover, every chamber of $X-X_{\beta}$ contains a tetrahedron such that lengthening along $\beta$ decreases volume.

One could say that Corollary 1.6 gives coarsely sharp conditions on when selective lengthening increases volume. Of course, if we used a finer triangulation, we could make finer statements about this.

We can get some weak partial results about the remaining two unfriendly configurations, those whose complement is either a single edge or a pair of incident edges. We will discuss this briefly at the end of $\S 5$. Our methods really do fail for these two configurations.

This paper has a companion computer program - a heavily documented and open-sourced graphical user interface - which the interested readers can download from

\section{http://www.math.brown.edu/ res/Java/CM2.tar}

The program does all the integer polynomial calculations, and also shows plots of the Cayley-Menger determinant and the various relevant directional derivatives, I discovered essentially everything in the paper using the program. 
Here is some speculation on related questions. Genevieve Walsh asked about results similar to Theorems 1.1 and 1.2 for other combinatorial types - e.g. the octahedron. One sensible constraint is that the maximum valence should be at most 5 , so that the combinatorial type can be realized as convex polyhedra with equilateral facets. There are explicit analogues of the Cayley-Menger determinant, which give volume formulas for other combinatorial types. See $[\mathrm{Sa}]$.

One might also ask about hyperbolic geometry versions of the results here. It seems that Schlafli's formula - see e.g. $[\mathbf{L}]$ - might be useful. It would be very nice to prove hyperbolic or spherical versions of these results, and then deduce the Euclidean results as limiting cases. I have no idea how to do this.

Just as Theorem 1.2 is a generalization of Theorem 1.1, I wonder if Theorem 1.4 and Corollary 1.6 have higher dimensional generalizations. It would be nice to find a conceptual proof of Theorem 1.4, because my techniques are unlikely to be feasible in higher dimensions.

I learned about Daryl Cooper's question during a lively semester program in computational geometry, topology, and dynamics at ICERM in Fall 2013. I thank Bob Connelly, Peter Doyle, Ramin Naimi, Igor Rivin, Sinai Robins, and Genevieve Walsh for interesting and helpful conversations about this problem, some at ICERM and some elsewhere. I would especially like to acknowledge some conversations with Peter Doyle which helped guide me towards the special 48-chamber decomposition of the space $X$. Peter made the great guess that the vertex and axis sums should be important in this edge-lengthening business. 


\section{Pseudo Tetrahedra}

\subsection{Normalized Pseudo Tetrahedra}

We say that a pseudo-tetrahedron is normalized if

$$
\sum_{i<j} d_{i j}=24
$$

Let $X_{24}$ denote the space of normalized pseudo-tetrahedra. The 48-partition of $X$ discussed in the introduction is the cone over a partition of $X_{24}$ into 48 5-simplices. We choose the normalization 24 because it is the smallest number we can choose which makes all these simplices integral. Since all the inequalities we stated in the introduction are homogeneous, it suffices to prove them on $X_{24}$.

There are 7 special points of $X_{24}$ :

- 3 of these points correspond to degenerate tetrahedra in which the points have collapsed in pairs.

- 4 of these points correspond to degenerate tetrahedra in which 3 of the points have collapsed to one.

The 7 vectors corresponding to these points are

- $A_{1}=(0,6,6,6,6,0)$.

- $A_{2}=(6,0,6,6,0,6)$.

- $A_{3}=(6,6,0,0,6,6)$.

- $B_{1}=(8,8,8,0,0,0)$.

- $B_{2}=(8,0,0,8,8,0)$.

- $B_{3}=(0,8,0,8,0,8)$.

- $B_{4}=(0,0,8,0,8,8)$.

We call these points extrema of $X_{24}$, for reasons which will become clear momentarily.

The following result is somewhat surprising, because the points above are all (degenerate) tetrahedra whereas $X_{24}$ certainly contains pseudo-tetrahedra which are not tetrahedra in any sense - e.g. $(6,3,3,3,3,6)$. 
Lemma 2.1 $X_{24}$ is the convex hull of the 7 extrema.

Proof: Let $C$ denote the convex hull of the extrema. Certainly $C \subset X$. For $k=1,2,3$, let $\boldsymbol{A}_{k}$ denote the convex hull of the list of 6 extrema obtained by omitting $A_{k}$. Each $\boldsymbol{A}_{k}$ is a 5-simplex, and $\boldsymbol{A}_{i} \cap \boldsymbol{A}_{j}$ is a 4 simplex. It is easy to check that $\boldsymbol{A}_{i}$ and $\boldsymbol{A}_{j}$ lie on opposite sides of the 4-plane containing their intersection.

Call a face of $\boldsymbol{A}_{j}$ free if it is not also a face of $\boldsymbol{A}_{j}$ for $j \neq i$. Otherwise, call the face bound. We have already exhibited 2 bound faces of each $\boldsymbol{A}_{j}$. For the remaining faces, we check that the barycenter of the face lies in $\partial X_{24}$. For instance, one of the barycenters of a free face of $\boldsymbol{A}_{1}$ is

$$
\left(A_{2}+A_{3}+B_{1}+B_{2}+B_{3}\right) / 5=(28,22,14,22,14,20) / 5 .
$$

In particular $d_{14}+d_{24}=d_{12}$. The barycenter condition implies that the entire free face lies in $\partial X$. This $\cup \boldsymbol{A}_{j}$ is a union of three 5-simplies, with pairwise disjoint interiors, whose boundary lies in $\partial X$. This is only possible if $X=\bigcup \boldsymbol{A}_{j}$. But $\bigcup \boldsymbol{A}_{j} \subset C$. Hence $C=X$.

The proof in the Lemma 2.1 shows that $X_{24}$ has a partition into 3 simplices. Let us consider the structure of $\boldsymbol{A}_{1}$. If we compute the axis sums of the labelings corresponding to the extrema, we find that these sums are all equal for the $B$-extrema, and (12)(34) has largest axis sum for $A_{2}$ and $A_{3}$. Thus $\boldsymbol{A}_{1}$ consists entirely of points whose largest axis sum is (12)(34). Similarly, $\boldsymbol{A}_{2}$ consists entirely of points whose largest axis sum is (13)(24), and $\boldsymbol{A}_{3}$ consists entirely of points whose largest axis sum is (14)(23).

We can also define the simplex $\boldsymbol{B}_{k}$, which is the convex hull of the list of 6 extrema obtained by omitting $B_{k}$. The same proof as above show that this gives a 4-partition of $X_{24}$. An analysis similar to what we did for the 3-partition shows that $\boldsymbol{B}_{k}$ consists of those points whose corresponding labelings of $K_{4}$ have smallest vertex sum at vertex $k$.

Remark: There is a beautiful lower-dimensional picture which gives a good feel for how the 3-partition and the 4-partition are related. One can think of a triangular bi-pyramid $T$ as the join of a triangle and a pair of points. Correspondingly, $T$ has a partition into 2 tetrahedra, and also a partition into 3 tetrahedra. This is the famous $2-3$ relation often discussed in connection with 3 -dimensional triangulations. The situation we have is a higher dimensional analogue of this. 


\subsection{The Common Refinement}

The space $X_{24}$ has a partition into 12 simplices, as follows: We define $\boldsymbol{C}_{i j}$ to be the convex hull of the point

$$
C=(4,4,4,4,4,4)
$$

and the list of 5 extrema obtained by omitting $A_{i}$ and $B_{j}$.

Lemma $2.2 C_{i j}=A_{i} \cap B_{j}$.

Proof: Note that

$$
C=\frac{1}{3} \sum A_{i}=\frac{1}{4} \sum B_{j}
$$

So that $C \subset \boldsymbol{A}_{i}$ and $C \subset \boldsymbol{B}_{j}$ for all $j$. Hence, all vertices of $\boldsymbol{C}_{i j}$ are contained in $\boldsymbol{A}_{i} \cap \boldsymbol{B}_{j}$. Hence $\boldsymbol{C}_{i j} \subset \boldsymbol{A}_{i} \cap \boldsymbol{B}_{j}$.

Next, we check that the barycenter of every face of $\boldsymbol{A}_{i j}$ lies in $\partial\left(\boldsymbol{A}_{i} \cap \boldsymbol{B}_{j}\right)$. By symmetry, it suffices to check this for $(i, j)=(1,4)$. Again by symmetry, it suffices to make the check for the face of which does not involve $C$ and for one additional face. The barycenter of the face not involving $C$ is the same as the one we computed in the proof of Lemma 2.1. This point must lie in both $\partial \boldsymbol{A}_{i}$ and $\partial \boldsymbol{B}_{4}$ because it lies in $\partial X_{24}$. One of the other barycenters is

$$
\left(A_{2}+A_{3}+B_{1}+B_{2}+C\right) / 5=(32,18,18,18,18,16) / 5 .
$$

This point lies in $\partial \boldsymbol{B}_{4}$ because the vertex sum at vertex 4 is the same as the vertex sum at vertex 3 . This check establishes what we want.

As in the proof of Lemma 2.1, the barycenter condition implies that every face of $\boldsymbol{C}_{i j}$ lies in $\partial\left(\boldsymbol{A}_{i} \cap \boldsymbol{B}_{j}\right)$. Since both sets are 5-dimensional convex polytopes, this situation is only possible if $\boldsymbol{C}_{i j}=\boldsymbol{A}_{i} \cap \boldsymbol{B}_{j}$.

Lemma 2.2 implies that $X_{24}$ has a partition into 12 simplices, namely $\boldsymbol{C}_{i j}$ for $i \in\{1,2,3\}$ and $j \in\{1,2,3,4\}$. The simplex $\boldsymbol{C}_{i j}$ consists of those labelings where the $i$ th vertex sum is smallest and the $j$ th axis sum is largest.

Remark: Once again, the picture for the bi-pyramid is useful here. The intersections of the 2-partition of the bi-pyramid with the 3-partition gives a 6-partition into smaller tetrahedra. Our situation here is a higher dimensional analogue of this. 


\subsection{The Final Partition}

The order 24 symmetric group $S_{4}$ acts on the space $X_{24}$ via label permutation. The even subgroup $A_{4}$ acts freely and transitively on our 12-partition. However, the full group $S_{5}$ does not act freely on the 12-partition. The stabilizer of each simplex is an order 2 subgroup. We will use this symmetry to facilitate the understanding of a refinement of the 12-partition into a 48partition. Basically, we cut each of the simplices into 4 symmetric pieces, again simplices. We do this for the simplex $\boldsymbol{C}_{11}$ and then use the $A_{4}$ symmetry to do it for the remaining simplices.

We let $A_{i j}=\left(A_{i}+A_{j}\right) / 2$ and likewise $B_{i j}=\left(B_{i}+B_{j}\right) / 2$. Also, we let $H(\cdot)$ stand the for convex hull. We introduce the 4 simplices

$$
\begin{aligned}
& \boldsymbol{D}_{1111}=\mathrm{H}\left(C, B_{2}, B_{34}, A_{23}, B_{3}, A_{2}\right) . \\
& \boldsymbol{D}_{1112}=\mathrm{H}\left(C, B_{2}, B_{34}, A_{23}, B_{3}, A_{3}\right) . \\
& \boldsymbol{D}_{1121}=\mathrm{H}\left(C, B_{2}, B_{34}, A_{23}, B_{4}, A_{2}\right) . \\
& \boldsymbol{D}_{1121}=\mathrm{H}\left(C, B_{2}, B_{34}, A_{23}, B_{4}, A_{3}\right) .
\end{aligned}
$$

Notice that only the last two vectors are changing. One can see direcly that $\bigcup \boldsymbol{D}_{11 i j}$ gives a partition of $\boldsymbol{D}_{11}$. What we are doing is subdividing the 3-simplex $H\left(B_{3}, B_{4}, A_{2}, A_{3}\right)$ into 4 symmetric pieces, and then taking the join with the segment $H\left(C, B_{2}\right)$. Figure 2.1 shows how to think about the subdivision of the tetrahedron.

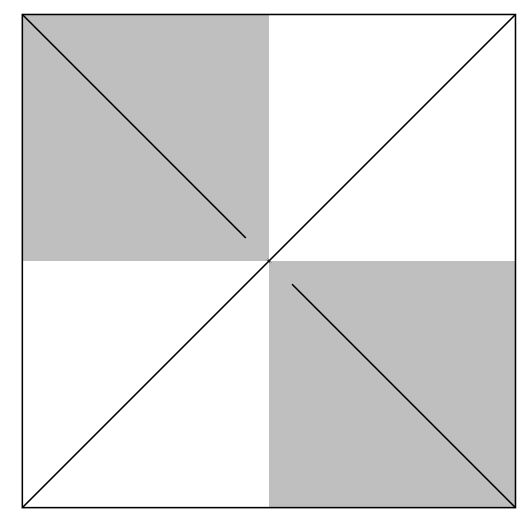

Figure 2.1: Top view of the 4-subdivision of a tetrahedron. 
As we mentioned above, we use the $A_{4}$ symmetry to promote our partition of $\boldsymbol{D}_{11}$ into a partition of all of $X_{24}$ into 48 simplices.

A direct calculation shows that the vertices of $\boldsymbol{D}_{11 i j}$ all satisfiy the inequalities associated to the decorations discussed in the introduction. Figure 2.2 shows the 4 decorations.
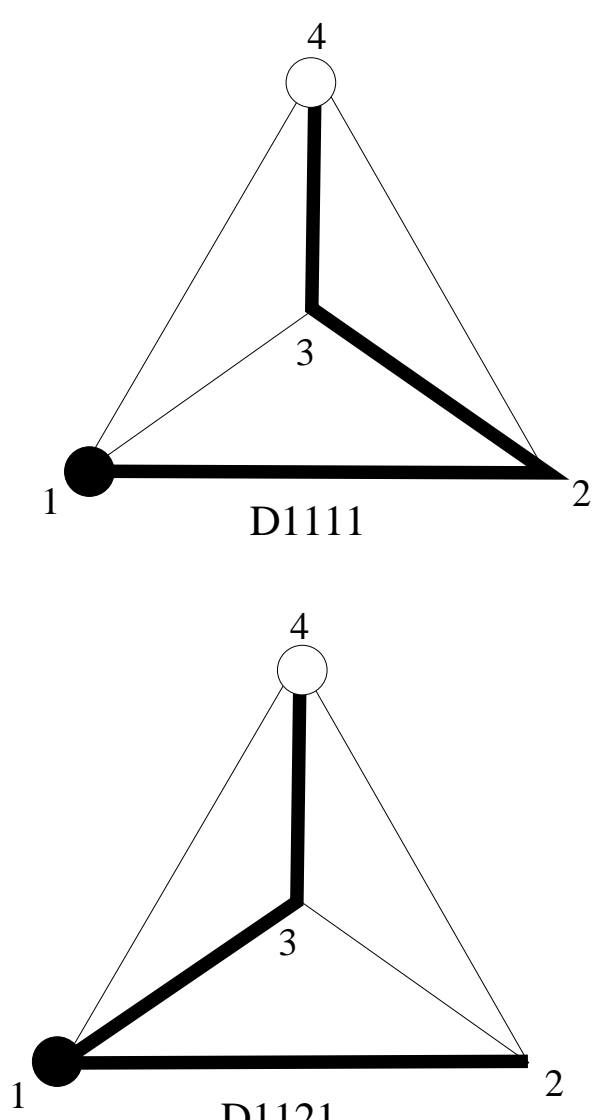

D1121
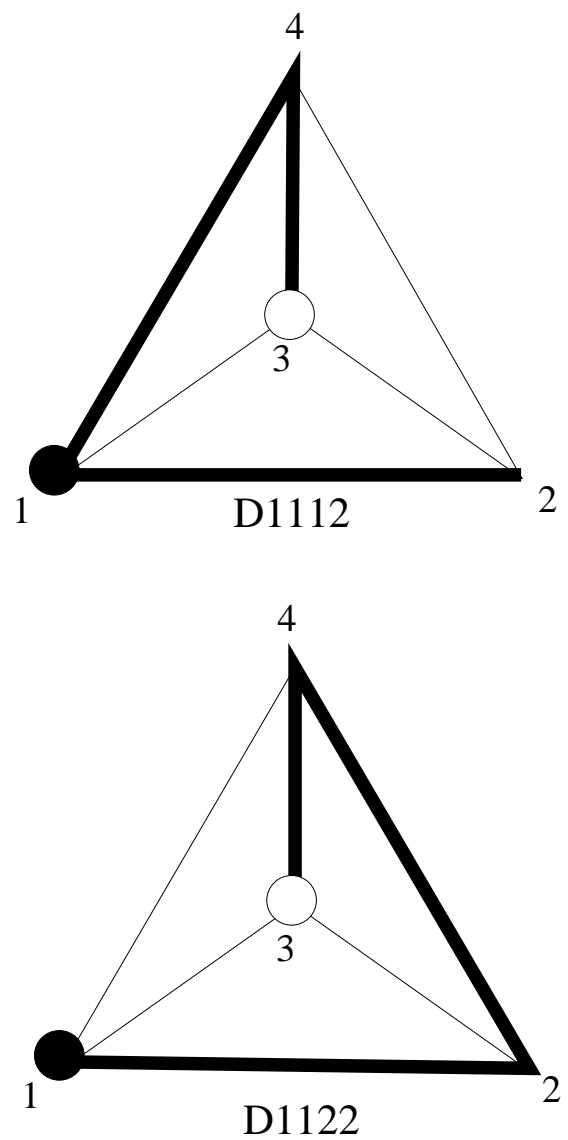

Figure 2.2: The decorations associated to $\boldsymbol{D}_{11 i j}$.

The partition discussed in the introduction restricts to a partition of $X_{24}$ into 48 convex polytopes. Each of these polytopes contains one of our sets $\boldsymbol{D}_{i j k \ell}$. But then the partition in the introduction must intersect $X_{48}$ precisely in the partition $\left\{\boldsymbol{D}_{i j k \ell}\right\}$ constructed here. By honogentity, each chamber in the partition from the introduction is the cone over some simplex in our partition here. This establishes the claim in the introduction that the chambers are linearly isomorphic to orthants. 


\subsection{Theorem 1.3 Modulo a Detail}

Let $f$ denote the Cayley-Menger determinant, as in Equation 1, and let $g$ be as in Equation 3. In $\S 3$ we will explain how we establish the following theorem:

Lemma $2.32 g-3 f$ and $3 g-2 f$ are non-negative on $\boldsymbol{D}_{11}$.

Corollary 2.4 $2 g-3 f$ and $3 g-2 f$ are non-negative on $X_{24}$.

Proof: The two functions $f$ and $g$ are invariant under the action of the alternating group $A_{4}$, and this action freely permutes the simplices in the 12-partition of $X_{24}$. Since we have non-negativity on one of these simplices, we get the non-negativity on all of them, by symmetry.

Lemma 2.5 Let $a$ and $b$ be constants. Then $b g+a f \geq 0$ on $X_{24}$ if and only if $b g+a f$ is a non-negative combination of the two functions in Lemma 2.3.

Proof: Recall that $C=(4,4,4,4,4,4)$. We compute that

$$
f(C)=3^{0} \times 2^{14}, \quad g(C)=3^{1} \times 2^{13} .
$$

This equation shows that $2 b+3 a \geq 0$.

We compute that

$$
f(6,3,3,3,3,6)=-3^{6} \times 2^{7}, \quad g(6,3,3,3,3,6)=-3^{5} \times 2^{8} .
$$

This equation shows that $-3 b-2 a \geq 0$.

Finally, by considering a degenerate tetrahedron with 3-fold symmetry and an equilateral triangle base, we see that it can happen that $f=0$ and $g>0$. This forces $b \geq 0$. Equation 11 then force $a \leq 0$. Our two inequalities above now confine $(a, b)$ to one half of a cone in the plane, and this cone is precisely the set of coeffients one can obtain by taking non-linear combinations of the ones in Lemma 2.3.

Combining the results in this section, with some basic arithmetic, and the fact that $\sum_{i<j} d_{i j}=24$, we see that Theorem 1.3 is true on $X_{24}$. But then, by homogeneity, Theorem 1.3 is true on all of $X$. 


\subsection{Proof of Theorem 1.1}

To prove Theorem 1.1, we find it convenient to work on the subset $X_{6} \subset X$ consisting of pseudo-tetrahedra $\left\{d_{i j}\right\}$ where $\sum_{i<j} d_{i j}=6$.

Lemma 2.6 On $X_{6}$ we have $g \geq 6 f$.

Proof: This is a special case of Theorem 1.3.

Rather than prove Theorem 1.1 for the unit lengthening of a tetrahedron, we will prove that the $t$-lenghening $\Delta_{t}$ of a tetrahedron $\Delta_{0} \in X_{6}$ satisfies the volume bound

$$
\frac{\operatorname{volume}\left(\Delta_{t}\right)}{\operatorname{volume}\left(\Delta_{0}\right)} \geq(1+t)^{3} \text {. }
$$

Theorem 1.1 follows from this result, and scaling.

Let $\phi_{t}: X \rightarrow X$ denote the flow defined by

$$
\phi_{t}\left(d_{i j}\right)=\left\{d_{i j}+t\right\}
$$

Let $D \in X_{6}$ represent $\Delta_{0}$. Let $D_{t}=\phi_{t}(D)$ and $F(t)=f\left(D_{t}\right)$.

Since $f$ is homogeneous of degree 6 and $g$ is homogeneous of degree 5 , Lemma 2.6 implies that

$$
\frac{d F}{d t} \geq \frac{6}{1+t} F(t)
$$

This shows immediately that $F$ is increasing. Suppose that $D_{t}$ fails to be tetrahedral for some $t>0$. This would force $F(t)=0$, contradicting the increase of $F$. This shows that $D_{t}$ is tetrahedral for all $t>0$.

Equation 14 can be rearranged as

$$
\frac{d}{d t} \log F \geq \frac{6}{1+t}
$$

Integrating both sides and then exponentiating, we get

$$
\frac{F(t)}{F(0)} \geq(1+t)^{6} \text {. }
$$

Taking square roots and using Equation 2, we get exactly Equation 12. 


\section{The Method of Positive Dominance}

In $\S 2$ we reduced Theorem 1.1 to Theorem 1.3. In this chapter we will explain our computational method for proving Theorem 1.3. The material in this chapter is taken mostly from my recent monograph $[\mathbf{S}]$, though it has been adapted to the present situation.

\subsection{Single Variable Case}

As a warmup, we consider the situation for polynomials in a single variable. Let

$$
P(x)=a_{0}+a_{1} x+\ldots+a_{n} x^{n}
$$

be a polynomial with real coefficients. Here we describe a method for showing that $P \geq 0$ on $[0,1]$,

Define

$$
A_{k}=a_{0}+\cdots+a_{k}
$$

We call $P$ weak positive dominant (or WPD for short) if $A_{k} \geq 0$ for all $k$.

Remark: To keep consistent with $[\mathbf{S}]$, we reserve the terminology positive dominant for the case $A_{k}>0$ for all $k$. However, in this paper we only care about weak positive dominance.

Lemma 3.1 If $P$ is weak positive dominant, then $P \geq 0$ on $[0,1]$.

Proof: The proof goes by induction on the degree of $P$. The case $\operatorname{deg}(P)=0$ follows from the fact that $a_{0}=A_{0} \geq 0$. Let $x \in[0,1]$. We have

$$
\begin{gathered}
P(x)=a_{0}+a_{1} x+x_{2} x^{2}+\cdots+a_{n} x^{n} \geq \\
a_{0} x+a_{1} x+a_{2} x^{2}+\cdots+a_{n} x^{n}= \\
x\left(A_{1}+a_{2} x+a_{3} x^{2}+\cdots a_{n} x^{n-1}\right)=x Q(x) \geq 0
\end{gathered}
$$

Here $Q(x)$ is weak positive dominant and has degree $n-1$.

Remark: The converse of Lemma 3.1 is generally false. We will give an example below. 
Given an interval $I \subset \boldsymbol{R}$, let $A_{I}$ be the affine and orientation preserving map which carries $[0,1]$ to $I$. We call the pair $(P, I)$ weak positive dominant if $P \circ A_{I}$ is WPD. If $(P, I)$ is WPD then $P \geq 0$ on $I$, by Lemma 3.1.

We say that a partition $[0,1]=I_{1} \cup \ldots \cup I_{n}$ is weak positive dominant with respect to $P$ if $\left(P, I_{k}\right)$ is WPD for each $k=1, \ldots, n$. For short, we will just say that $P$ has a weak positive dominant partition. If $P$ has a WPD partition, then $P \geq 0$ on $[0,1]$.

Example: The polynomial

$$
P(x)=3-4 x+2 x^{2}
$$

is not WPD but satisfies $P(x) \geq 1$ for all $x \in \boldsymbol{R}$. Consider the partition $[0,1]=I_{1} \cup I_{2}$, where $I_{1}=[0,1 / 2]$ and $I_{2}=[1 / 2,1]$. The corresponding affine maps are

$$
A_{1}(x)=x / 2 ; \quad A_{2}(x)=x / 2+1 / 2 .
$$

We compute

$$
P \circ A_{1}(x)=3-2 x+x^{2} / 2, \quad P \circ A_{2}(x)=3 / 2-x+x^{2} / 2 .
$$

Both of these polynomials are WPD. Hence $P$ has a WPD partition.

Divide-and-Conquer Algorithm: If $P \geq 0$ on $[0,1]$, we can try to find a WPD partition using a divide-and-conquer algorithm. The algorithm works like this.

1. Start with a list LIST of intervals. Initially LIST consists only of $[0,1]$.

2. Let $I$ be the last interval on LIST. We delete $I$ from LIST and then test whether $(P, I)$ is weak positive dominant.

3. Suppose $(P, I)$ is weak positive dominant. We go back to Step 2 if LIST is nonempty and otherwise halt.

4. Suppose $(P, I)$ is not weak positive dominant. We append to LIST the two intervals obtained from cutting $I$ in half, then go back to Step 2.

If the algorithm halts, then (assuming that the calculations are done exactly) we have a proof that $P \geq 0$ on $[0,1]$. 


\subsection{The General Case}

Now we go to the higher dimensional case. We consider real polynomials in the variables $x_{1}, \ldots, x_{k}$. Given a multi-index $I=\left(i_{1}, \ldots, i_{k}\right) \in(\boldsymbol{N} \cup\{0\})^{k}$ we let

$$
x^{I}=x_{1}^{i_{1}} \ldots x_{k}^{i_{k}} .
$$

Any polynomial $F \in \boldsymbol{R}\left[x_{1}, \ldots, x_{k}\right]$ can be written succinctly as

$$
F=\sum A_{I} X^{I}, \quad A_{I} \in \boldsymbol{R} .
$$

If $I^{\prime}=\left(i_{1}^{\prime}, \ldots, i_{k}^{\prime}\right)$ we write $I^{\prime} \leq I$ if $i_{j}^{\prime} \leq i_{j}$ for all $j=1, \ldots, k$. We call $F$ weak positive dominant if

$$
\sum_{I^{\prime} \leq I} A_{I^{\prime}} \geq 0 \quad \forall I
$$

Lemma 3.2 If $P$ is weak positive dominant then $P \geq 0$ on $[0,1]^{k}$.

Proof: The 1 variable case is Lemma 3.1. In general, we write

$$
P=f_{0}+f_{1} x_{k}+\ldots+f_{m} x_{k}^{m}, \quad f_{j} \in \boldsymbol{R}\left[x_{1}, \ldots, x_{k-1}\right] .
$$

Let $P_{j}=f_{0}+\ldots+f_{j}$. Since $P$ is weak positive dominant, we get that $P_{j}$ is weak positive dominant for all $j$. By induction on $k$, we get $P_{j} \geq 0$ on $(0,1)^{k-1}$. But now, if we hold $x_{1}, \ldots, x_{k-1}$ fixed and let $t=x_{k}$ vary, the polynomial $g(t)=P\left(x_{1}, \ldots, x_{k-1}, t\right)$ is weak positive dominant.. Hence, by Lemma 3.1 , we get $g \geq 0$ on $[0,1]$. Hence $P \geq 0$ on $[0,1]^{k}$.

We can perform the same kind of divide-and-conquer algorithm as in the 1-dimensional case. We always take our domain to be $[0,1]^{k}$. Let $P$ be a polynomial. We are going to describe our subdivision in terms of what it does to the polynomials rather than what it does to the domain.

We first define the maps

$$
\begin{gathered}
A_{j}\left(x_{1}, \ldots, x_{k}\right)=\left(x_{1}, . . x_{j-1} ., \frac{x_{j}}{2}, x_{j+1} \ldots, x_{k}\right) . \\
B_{j}\left(x_{1}, \ldots, x_{j}, \ldots, x_{k}\right)=\left(x_{1}, \ldots, x_{j-1}, 1-x_{j}, x_{j+1} \ldots, x_{k}\right) .
\end{gathered}
$$

We define the $j$ th subdivision of $P$ to be the set

$$
\left\{P_{j 1}, P_{j 2}\right\}=\left\{P \circ A_{j}, P \circ B_{j} \circ A_{j}\right\} .
$$


Lemma $3.3 P \leq 0$ on $[0,1]^{k}$ if and only if $P_{j 1} \geq 0$ and $P_{j 2} \geq 0$ on $[0,1]^{k}$.

Proof: By symmetry, it suffices to take $j=1$. Define

$$
[0,1]_{1}^{k}=[0,1 / 2] \times[0,1]^{k-1}, \quad[0,1]_{2}^{k}=[1 / 2,1] \times[0,1]^{k-1} .
$$

Note that

$$
A_{1}\left([0,1]^{k}\right)=[0,1]_{1}^{k}, \quad B_{1} \circ A_{1}\left([0,1]^{k}\right)=[0,1]_{2}^{k} .
$$

Therefore, $P \geq 0$ on $[0,1]_{1}^{k}$ if and only if $P_{j 1} \geq 0$ on $[0,1]^{k}$. Likewise $P \geq 0$ on $[0,1]_{2}^{k}$ if and only if if $P_{j 2} \geq 0$ on $[0,1]^{k}$.

Say that a marker is a non-negative integer vector in $\boldsymbol{R}^{k}$. Say that the youngest entry in the the marker is the first minimum entry going from left

to right. The successor of a marker is the marker obtained by adding one to the youngest entry. For instance, the successor of $(2,2,1,1,1)$ is $(2,2,2,1,1)$. Let $\mu_{+}$denote the successor of $\mu$.

We say that a marked polynomial is a pair $(P, \mu)$, where $P$ is a polynomial and $\mu$ is a marker. Let $j$ be the position of the youngest entry of $\mu$. We define the subdivision of $(P, \mu)$ to be the pair

$$
\left\{\left(P_{j 1}, \mu_{+},\left(P_{j 2}, \mu_{-}\right)\right\} .\right.
$$

Geometrically, we are cutting the domain in half along the longest side, and using a particular rule to break ties when they occur.

\section{Divide-and-Conquer Algorithm:}

1. Start with a list LIST of marked polynomials. Initially, LIST consists only of the marked polynomial $(P,(0, \ldots, 0))$.

2. Let $(Q, \mu)$ be the last element of LIST. We delete $(Q, \mu)$ from LIST and test whether $Q$ is weak positive dominant.

3. Suppose $Q$ is weak positive dominant. we go back to Step 2 if LIST is not empty. Otherwise, we halt.

4. Suppose $Q$ is not weak positive dominant. we append to LIST the two marked polynomials in the subdivision of $(Q, \mu)$ and then go to Step 2.

We call $P$ Recursively Weak Positive Dominant or (RWPD) if the divide and conquer algorithm halts for $P$. If $P$ is RWPD then $P \geq 0$ on $[0,1]^{k}$. This is a consequence of Lemma 3.3 and induction on the number of steps taken in the algorithm. 


\subsection{From Cubes to Simplices}

So far we have been talking about showing that polynomials are non-negative on the unit cube $[0,1]^{k}$. But, we really want to show that the polynomials of interest to us, namely those from Lemma 2.3, are non-negative on the simplex $\boldsymbol{D}_{11}$. In this section, we explain how this is done. We set $k=5$ and use coordinates $(a, b, c, d, e)$ on $\boldsymbol{R}^{5}$.

Cube To Standard Simplex: Let $S_{5} \subset \boldsymbol{R}^{5}$ denote the simplex

$$
\{(a, b, c, d, e) \mid 1 \geq a \geq b \geq c \geq d \geq e \geq 0\}
$$

We call $S_{5}$ the standard simplex, though actually we won't use this terminology after this section. There is a polynomial surjective map from $[0,1]^{5}$ to $S_{5}$ :

$$
U(a, b, c, d, e)=(a, a b, a b c, a b c d, a b c d e) .
$$

Standard Simplex to Regular Simplex: Let $\Delta_{6} \subset \boldsymbol{R}^{6}$ denote the regular 5-simplex in $\boldsymbol{R}^{6}$ consisting of the convex hull of the standard basis vectors. That is, $\Delta_{6}$ consists of points $\left(x_{1}, \ldots, x_{6}\right)$ such that $x_{j} \geq 0$ for all $j$ and $\sum x_{j}=1$. There is an affine isomorphism from $S_{5}$ to $\Delta_{6}$ :

$$
V(a, b, c, d, e)=(1-a, a-b, b-c, c-d, d-e, e) .
$$

The easiest way to see that this works is to check it on the vertices of $S_{5}$.

Regular Simplex to General Simplex: Let $\Sigma$ denote a 5-simplex in $\boldsymbol{R}^{6}$. We can think of $\Sigma$ as a $6 \times 6$ matrix whose 6 columns are the vertices of $\Sigma$. Call this matrix $W_{\Sigma}$. The map $W_{\Sigma}$ gives an affine isomorphism from $\Delta_{6}$ to $\Sigma$.

Note that the composition

$$
Z_{\Sigma}=W_{\Sigma} \circ V \circ U:[0,1]^{5} \rightarrow \Sigma
$$

is a surjective rational map. Given a polynomial $P$ and a simplex $\Sigma \subset \boldsymbol{R}^{5}$, we define the new polynomial

$$
P_{\Sigma}=P \circ Z_{\Sigma}
$$

By construction, $P \geq 0$ on $\Sigma$ provided that $P_{\Sigma}$ is RWPD. 


\subsection{Proof of Lemma 2.3}

Let $P$ and $Q$ be the two polynomials from Lemma 2.3 and let $\Sigma=\boldsymbol{D}_{11}$, the simplex from Lemma 2.3.

Lemma 3.4 $P_{\Sigma}$ and $Q_{\Sigma}$ are both $R W P D$.

Proof: We prove Lemma 3.4 simply by coding all the algebra in sight into a Java program and running it. In the next chapter we discuss the implementations of our calculations. For the function $P_{\Sigma}$ the algorithm takes 7455 steps and runs in about 62 minutes on my 2012 Macbook pro. For the function $Q_{\Sigma}$, the algorithm takes 1173 steps and runs in about 6 minutes.

This proves Lemma 2.3.

Remarks: For a given function, the number of steps in the algorithm would be the same on any perfectly running computer, but of course the time would vary. The number of steps looks large, but one has to remember that we are running the algorithm on a 5 dimensional cube. Note that $\left(2^{3}\right)^{5}=32768$, so we are making an average of less than 3 subdivisions in each coordinate direction. 


\section{Implementation}

We implement our calculations in Java. Here we describe the salient features of the code.

\subsection{Formulas for the Main Functions}

The reader can find explicit formulas for the Cayley-Menger determinant $f$ and the partial derivatives $\partial_{j} f$ for $j=1,2,3,4,5,6$ in the file DataCM.java. This is one of the files in the directory you get when you download my program from

\section{http://www.math.brown.edu/ res/Java/CM2.tar}

Aside from the formulas for $f$ and its partial derivatives, which we derived using Mathematica $[\mathbf{W}]$, the rest of the program is self-contained, in that all the calculations for the paper are done there. In an earlier version of the paper and program, I implemented some of the calculations in Mathematica, but that is no longer the case.

\subsection{General Features}

The program is written entirely in Java. It has several useful features.

- The user can see all the code.

- The user can see all the decorations corresponding to the 48-simplex partition.

- The user can run his/her own experiments, testing various linear combinations of $f$ and its partial derivatives for positivity.

- While running, the program has a documentation feature, so that the user can learn about practically every facet of the program by reading the text.

- The program has a debugging tool which allows one to see that the critical formulas really are correct.

Aside from the debugging and the experiment modes, all the calculations having to do with the proofs are done with exact integer arithmetic. 


\subsection{Data Structures}

Here we describe the special data structures used by our program.

BigIntegers: The BigInteger class in Java is designed to do arbitrary digit arithmetic. In practice this means that one can do arithmetic with integers which have thousands of digits. In our case, we never get integers with more than, say, 20 digits. So, we are well inside the working power of the language.

Monomials: For us, a MonoN is a tuple $\left[C,\left(e_{1}, \ldots, e_{n}\right)\right]$. Here $C$ is a BigInteger and $e_{1}, \ldots, e_{n}$ are non-negative integers. This expression has the following meaning.

$$
\left[C,\left(e_{1}, \ldots, e_{n}\right)\right]=C \prod_{i=1}^{5} x_{n}^{e_{n}} .
$$

We only implement this class for $N=5$ and $N=6$, and we found it convenient to define separate classes for each case.

Polynomials: A poly $N$ is a finite list of monomials. In practice we allow for 50000 (Mono6)s and 20000 (Mono5)s. For a Poly5, we have a universal degree bound $e_{j} \leq 6$ for all $j$. We don't keep track of the degree bound for a Poly6, but the universal bound is around 6 as well.

\subsection{Poly6 Arithmetic}

We use the Poly6 class to implement the maps discussed in $§ 3.3$. The functions $f$ and $\partial_{i} f$ are stored as lists of integers which the program readily converts into (Poly6)s. Starting with a Poly6 P, which is some integer combination of $f$ and its partial derivatives, and an integer simplex $\Sigma \subset X_{24}$, we compute the polynomial $P_{\Sigma}$. The implementation is straightforward, and basically is built out of polynomial addition and multiplication. The routines are contained in the fairly well documented file Poly6.java.

In principle, the function $P_{\Sigma}$ could be precomputed using Mathematica, since we just need to load in the formula once, and then process it. Indeed, an earlier version of the program did this. However, since we wanted to test many functions, we didn't want to repeatedly go into Mathematica and save the output to our Java files. My point is that the critical polynmomial algebra we do is the implementation of the Positive Dominance Algorithm. 


\subsection{Poly5 Arithmetic}

Here we describe the basic operations we perform on polynomials. The main point is to implement the Positive Dominance Algorithm.

Rotation: The 1 -rotation of $\left[C,\left(e_{1}, \ldots, e_{5}\right)\right]$ is $\left[C,\left(e_{5}, e_{1}, e_{2}, e_{3}, e_{4}\right)\right]$. The $k$ rotation is obtained by applying the 1 -rotation $k$ times. The $k$-rotation of a polyomial is simply the list of $k$-rotations of its monomials. The $k$-rotation $R_{k} P$ of a polynomial $P$ is simply the composition of $P$ with some cyclic permutation of the coordinates.

If we have some operation $Z$ which does something to the first coordinates of the monomials of $P$, the operation $R_{k-1} Z R_{-k+1}$ does the same thing to the $k$ th coordinate. We use this trick so that we just have to implement our main routines for the first coordinate.

Dilation: We call the operation in Equation 25 dilation. We only implement dilation for the first variable. Given a polynomial $P$, the two polynomials from Equation 25 are not necessarily integer polynomials. Their coefficients are dyadic rationals: Expressions of the form $p / 2^{e_{1}}$. We let $E=\max e_{j} \leq 6$. Where the expression is taken over all monomials. To get an integer polynomial, we use $2^{E} P_{11}$ and $2^{E} P_{12}$ instead of $P_{11}$ and $P_{12}$.

Reflection: Here reflection is the operation of replacing the polynomial $P$ with the polynomial $P \circ B_{j}$. When $j$, the new polynomial is $P(1-a, b, c, d, e)$. We only implement the reflection operation for the first variable. The reflection operation is the rate limiting step in our program, so we explain the implementation carefully. We first create a $7 \times 7 \times 7 \times 7 \times 7$ array $\beta$ of BigIntegers. For each term $\left[C,\left(e_{1}, \ldots, e_{5}\right)\right]$ of the polynomial we perform the following: We let $\tau$ be the $\left(e_{1}\right)$ st row of Pascal's triangle, with the sign switched on the even terms. For instance, rows 0 and 1 and 2 are (0) and $(-1,1)$ and $(1,-2,-1)$ respectively. We then make the substitution

$$
\beta\left[j, e_{2}, e_{3}, e_{4}, e_{5}\right]=\beta\left[j, e_{2}, e_{3}, e_{4}, e_{5}\right]+C \tau(j), \quad j=0, \ldots, e_{1} .
$$

Again, we always have $e_{1} \in\{0, \ldots, 6\}$.

When we are done, we convert our array $\beta$ back into a polynomial by including the monomial $\left[C,\left(e_{1}, \ldots, e_{5}\right)\right]$ iff the final value of $\beta\left(e_{1}, \ldots, e_{5}\right)$ is $C$. The resulting polynomial is the reflection of $P$. 
Subdivision: The polynomial $P_{k 1}$ is obtained by the following operations:

1. Let $Q$ be the $(-k)$ th rotation of $P$.

2. Let $R$ be the dilation of $Q$.

3. Let $S$ be the $(k)$ th rotation of $R$.

4. Return $S$.

The polynomial $P_{k 2}$ is obtained by the following operations:

1. Let $Q$ be the $(-k)$ th rotation of $P$.

2. Let $Q^{*}$ be the reflection of $Q$.

3. Let $R$ be the dilation of $Q^{*}$.

4. Let $S$ be the $(k)$ th rotation of $R$.

5. Return $S$.

In the case of $P_{12}$ it is very important that steps 2 and 3 are not interchanged.

\subsection{Test for Weak Positive Dominance}

Building on the notation of Equation 34, we write

$$
\left[C,\left(e_{1}, \ldots, e_{5}\right)\right] \preceq\left(i_{1}, \ldots, i_{5}\right)
$$

if and only if $e_{j} \leq i_{j}$ for all $j$. Let $E$ be the smallest multi-index so that $\left[C,\left(e_{1}, \ldots, e_{5}\right)\right] \preceq E$ for all terms. We do the following loop.

for $\left(i_{1}=0 ; i_{1} \leq E_{1} ;++i_{1}\right)\{$

for $\left(i_{2}=0 ; i_{2} \leq E_{2} ;++i_{2}\right)\{$

for $\left(i_{3}=0 ; i_{3} \leq E_{3} ;++i_{3}\right)\{$

for $\left(i_{4}=0 ; i_{4} \leq E_{4} ;++i_{4}\right)\{$

for $\left(i_{5}=0 ; i_{5} \leq E_{5} ;++i_{5}\right)\{$

Sum the coefficients of all terms $\left[\left(c,\left(e_{1}, \ldots, e_{5}\right)\right] \preceq\left(i_{1}, \ldots, i_{5}\right)\right.$. \}\}\}\}\}.

We return "false" if we ever get a negative total sum. Otherwise we return "true", indicating that the polynomial is weak positive dominant. This procedure is probably rather far from being optimal, but it is quite simple. 


\subsection{Test for Negativity}

At each step of the WPDA, we perform an additional test. We check whether or not the current polynomial is negative at the origin. If the current polynomial is negative at the origin, we terminate the algorithm because we have a proof that the original polynomial is not non-negative on the given simplex. In this way, our algorithm typically halts either with a proof of non-negativity or a proof that some negative values exist.

There is the theoretical possibility that we could encounter a non-negative function that is not recursively weak positive dominant. For instance, the polynomial $P(x, y)=(x-y)^{2}$ is non-negative on the unit cube in any dimenson greater than 2, but not RWPD. Fortunately, we do not encounter polynomials like this in practice. So, in all cases, our algorithm terminates with a definite conclusion.

\subsection{Anti-Certification}

Supposing that $\beta$ is some subset of edges, our results also make statements about the chambers of the space $X$ which do not belong to the union $X_{\beta}$. Let $Y$ be such a chamber. On $Y$ we want to show that it can happen that $f>0$ and $d f<0$. here $d f$ is the directional derivative $D_{\beta} f$. In this section, we explain how we do this rigorously.

We sample random points in $Y_{24}$ until we find a candidate point $p \in Y_{24}$ such that (according to floating point calculations, it appears that) $f(p)>0$ and $d f(p)<0$. We replace $p$ by a point $p^{*} \in Y \cap \boldsymbol{Z}^{6}$ in such a way that $p$ and $p^{*}$ nearly lie on the same line through the origin. We then show that $f(p *)>0$ and $d f\left(p^{*}\right)<0$ using exact integer calculations.

It doesn't matter how we produce $p^{*}$,and it also doesn't matter that $p$ and $p^{*}$ nearly lie on the same line through the origin, but this property makes it likely that $f\left(p^{*}\right)>0$ and $d f\left(p^{*}\right)<0$. Even though these details don't matter from a logical standpoint, it seems worth explaining how we get $p^{*}$. The point $p$ has the form $L(q)$, where $L$ is an integer linear map taking the standard simplex $\sum x_{i}=1$ to $Y_{24}$. We then replace $q$ by the point

$$
q^{*}=\text { floor }\left(10^{10} q\right)
$$

and set $p^{*}=L\left(q^{*}\right)$. This does the job for us. 


\section{$5 \quad$ Selective Lengthening}

\subsection{A Single Edge}

The goal of this chapter is to prove Theorem 1.4, and to establish all the supplementary facts mentioned after we stated Theorem 1.4. We just go through the cases one at a time.

Let $\beta$ denote a single edge. Let $X_{\beta}$ be the union of 12 chambers $X_{D}$ such that $\beta \not \subset D$ and the black vertex of $D$ is an endpoint of $\beta$. Figure 5.1 shows 3 representative examples, corresponding to the simplices listed below. dThe edge $\beta$ is drawn in grey.
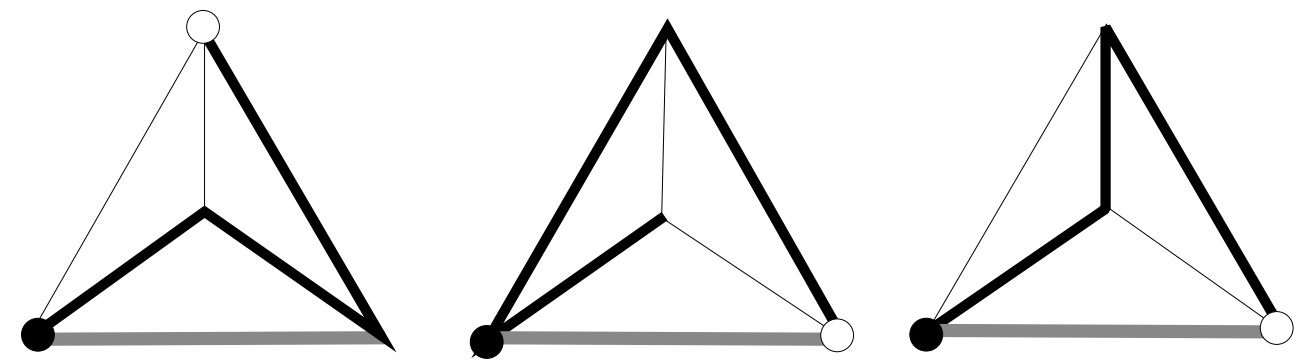

Figure 5.1: Three of the 12 decorations for $X_{\beta}$.

First of all, we use the anti-certification algorithm discussed in $\S 4.8$ to show that any chamber of $X-X_{\beta}$ has a point where $f>0$ and $g<0$. To save words, we will say below that we anti-certify the chambers not in $X_{\beta}$.

Now we turn to $X_{\beta}$. The 12 chambers intersect the normalized space $X_{24}$ in 12 simplices. If we pick $\beta=\left\{e_{12}\right\}$, then 3 of these simplices are given by

$$
\begin{aligned}
& (4,4,4,4,4,4),(0,8,0,8,0,8),(4,0,4,4,8,4) \\
& (3,6,3,3,6,3),(8,0,0,8,8,0),(0,6,6,6,6,0)
\end{aligned}
$$

and

$$
\begin{aligned}
& (4,4,4,4,4,4),(0,8,0,8,0,8),(4,0,4,4,8,4) \\
& (3,6,3,3,6,3),(0,0,8,0,8,8),(0,6,6,6,6,0)
\end{aligned}
$$

and

$$
\begin{aligned}
& (4,4,4,4,4,4),(0,8,0,8,0,8),(4,0,4,4,8,4) \\
& (3,6,3,3,6,3),(0,0,8,0,8,8),(6,6,0,0,6,6)
\end{aligned}
$$


The remaining simplices are images of these under the action of the subgroup of $S_{4}$ which stabilizes $e_{12}$.

We work with the functions

$$
P=g, \quad Q=12 g-f .
$$

Again $g=D_{\beta} f$. This function depends on $\beta$, of course. These are multiples of the ones mentioned above. We use the Method of Positive Dominance to check that both these functions are non-negative on the 3 simplices above. By symmetry, $P$ and $Q$ are non-negative on the intersection of $X_{24}$ with $X_{\beta}$. But then, by homogeneity, they are non-negative on $X_{\beta}$. For $P$, the number of steps taken for the three simplices is $421,421,427$ respectively. For $Q$, the number of steps taken for the three simplices is 457, 469, 617 respectively.

Our calculations show that the cone $y=0$ and $y>x / 12$ contains all points of the form $(f, g)$ when this pair of functions is evaluated on $X_{\beta}$. To show that $\left(A_{\beta}, B_{\beta}\right)=(0,2)$, we just need to see that there is no smaller cone which has this property. In other words, if we tilt the two lines bounding the cone inward, so to speak, the lines will cross points of the image. We deal with the two lines in turn.

One of the edges of $X_{\beta}$ has endpoints $(0,6,6,6,6,0)$ and $(3,6,3,3,6,3)$. Consider the point

$$
\Omega_{t}=(1-t)(0,6,6,6,6,0)+t(3,6,3,3,6,3) .
$$

We compute that

$$
g\left(\Omega_{t}\right)+t f\left(\Omega_{t}\right)=-342144 t^{3}+O\left(t^{4}\right) .
$$

This quantity is negative for all sufficiently small $t>0$. Geometrically, any line of negative slope through the origin, sufficiently close to the horizontal, contains points of $(f, g)$ on both sides.

Another edge of $X_{\beta}$ has endpoints $(8,0,0,8,8,0)$ and $(4,4,4,4,4,4)$. We restrict our functions to the point

$$
\Psi_{t}=\left(1-t^{2}\right)(8,0,0,8,8,0)+t^{2}(4,4,4,4,4,4) .
$$

a point which lies in an edge of $X_{\beta}$ for small $t$. Using Mathematica, we compute that Let

$$
(12-t) g\left(\Psi_{t}\right)-f\left(\Psi_{t}\right)=-57344 t^{5}+O\left(t^{6}\right) .
$$

This function is negative for all sufficiently small $t>0$. Geometrically, if we take the line of slope $1 / 12$ through the origin and increase its slope by any small positive amount, points of $(f, g)$ will lie on either side of the line. 


\subsection{A Pair of Incident Edges}

Let $\beta$ be a pair of incident edges. Let $X_{\beta}$ is the set of 4 chambers $X_{D}$ such that $\beta$ is disjoint from the outer two edges of $D$ and the black dot is the common endpoint of the two edges of $\beta$. Put another way, the shortest vertex-sum occurs at the vertex incident to the two edges of $\beta$, and the pair of opposites with the largest axis sum is disjoint from $\beta$ except at the endpoints. There are 4 such chambers. Figure 5.2 shows the decorations corresponding to 2 of the chambers. These decorations correspond to the simplices listed below. The other 2 are the images of these two under the element of $K_{4}$ which stabilizes $\beta$.
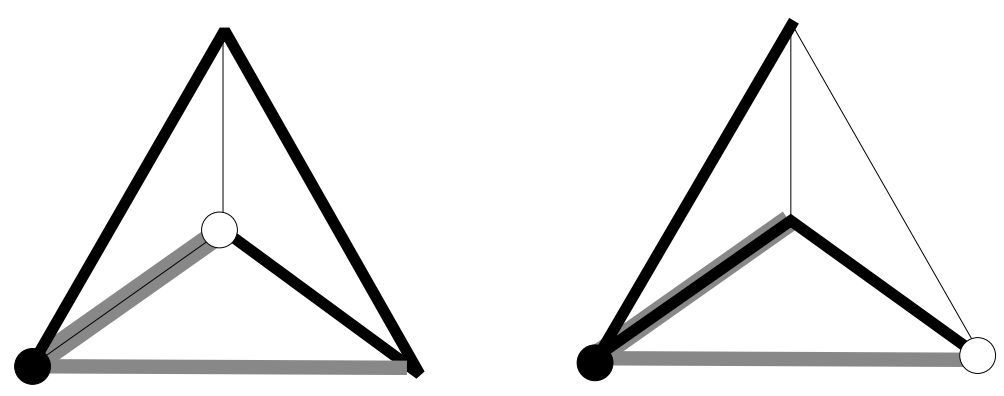

Figure 5.2: Two of four the decorations for $X_{\beta}$.

We take $\beta=\left\{e_{12}, e_{13}\right\}$. First, we anti-certify all the chambers not in $X_{\beta}$. Now we turn to $X_{\beta}$.

Two of the chambers of $X_{\beta}$ intersect $X_{24}$ in the simplices

$$
\begin{aligned}
& (4,4,4,4,4,4),(0,8,0,8,0,8),(4,0,4,4,8,4) \\
& (3,3,6,6,3,3),(8,0,0,8,8,0),(0,6,6,6,6,0)
\end{aligned}
$$

and

$$
\begin{aligned}
& (4,4,4,4,4,4),(0,8,0,8,0,8),(4,0,4,4,8,4) \\
& (3,3,6,6,3,3),(0,8,0,8,0,8),(0,6,6,6,6,0)
\end{aligned}
$$

The other two simplices are images of these under the order 2 subgroup of $S_{4}$ which stabilizes $\beta$.

We work with the functions

$$
P=g \quad Q=2 g-f
$$


The PDA takes 421 steps to certify that $P \geq 0$ on each simplex, and 479 steps to certify that $Q \geq 0$ on each simplex. These calculations show that $A_{\text {beta }} \leq 0$ and $B_{\beta} \geq 12$. Now we prove equality.

Let $V_{1}, \ldots, V_{6}$ be the 6 vertices of the first simplex listed above. Call this simplex $\Sigma$. Define

$$
\Omega_{t}=\left(1-t-t^{2}\right) V_{5}+t V_{2}+t^{2} V_{3} \in \Sigma .
$$

We compute that

$$
g\left(\Omega_{t}\right)+t\left(\Omega_{t}\right)=-2097152 t^{7}+O\left(t^{8}\right) .
$$

We also compute that

$$
(2-t) v f\left(V_{1}\right)-f\left(V_{1}\right)=-8192 t .
$$

The same argument as in the previous section shows that $\left(A_{\beta}, B_{\beta}\right)=(0,12)$.

\subsection{A Pair of Opposite Edges}

Let $\beta$ be a pair of opposite edges. Let $X_{\beta}$ be the set of 32 chambers $X_{D}$ such that $\beta \not \subset D$. In other words, the largest axis sum does not occur at $\beta$.

We take $\beta=\left\{e_{12}, e_{34}\right.$. First, we anti-certify the chambers of $X-X_{\beta}$.

Using the symmetry of the permutation group, it suffices to consider the 4 chambers which intersect $X_{24}$ in the following simplices.

$$
\begin{aligned}
& (4,4,4,4,4,4),(0,8,0,8,0,8),(4,0,4,4,8,4) \\
& (3,6,3,3,6,3),(8,0,0,8,8,0),(0,6,6,6,6,0)
\end{aligned}
$$

and

$$
\begin{aligned}
& (4,4,4,4,4,4),(0,8,0,8,0,8),(4,0,4,4,8,4) \\
& (3,6,3,3,6,3),(8,0,0,8,8,0),(6,6,0,0,6,6)
\end{aligned}
$$

and

$$
\begin{aligned}
& (4,4,4,4,4,4),(8,8,8,0,0,0),(4,0,4,4,8,4) \\
& (3,6,3,3,6,3),(8,0,0,8,8,0),(0,6,6,6,6,0)
\end{aligned}
$$

and

$$
\begin{aligned}
& (4,4,4,4,4,4),(8,8,8,0,0,0),(4,0,4,4,8,4) \\
& (3,6,3,3,6,3),(8,0,0,8,8,0),(6,6,0,0,6,6)
\end{aligned}
$$

Figure 5.3 shows the corresponding decorations. 

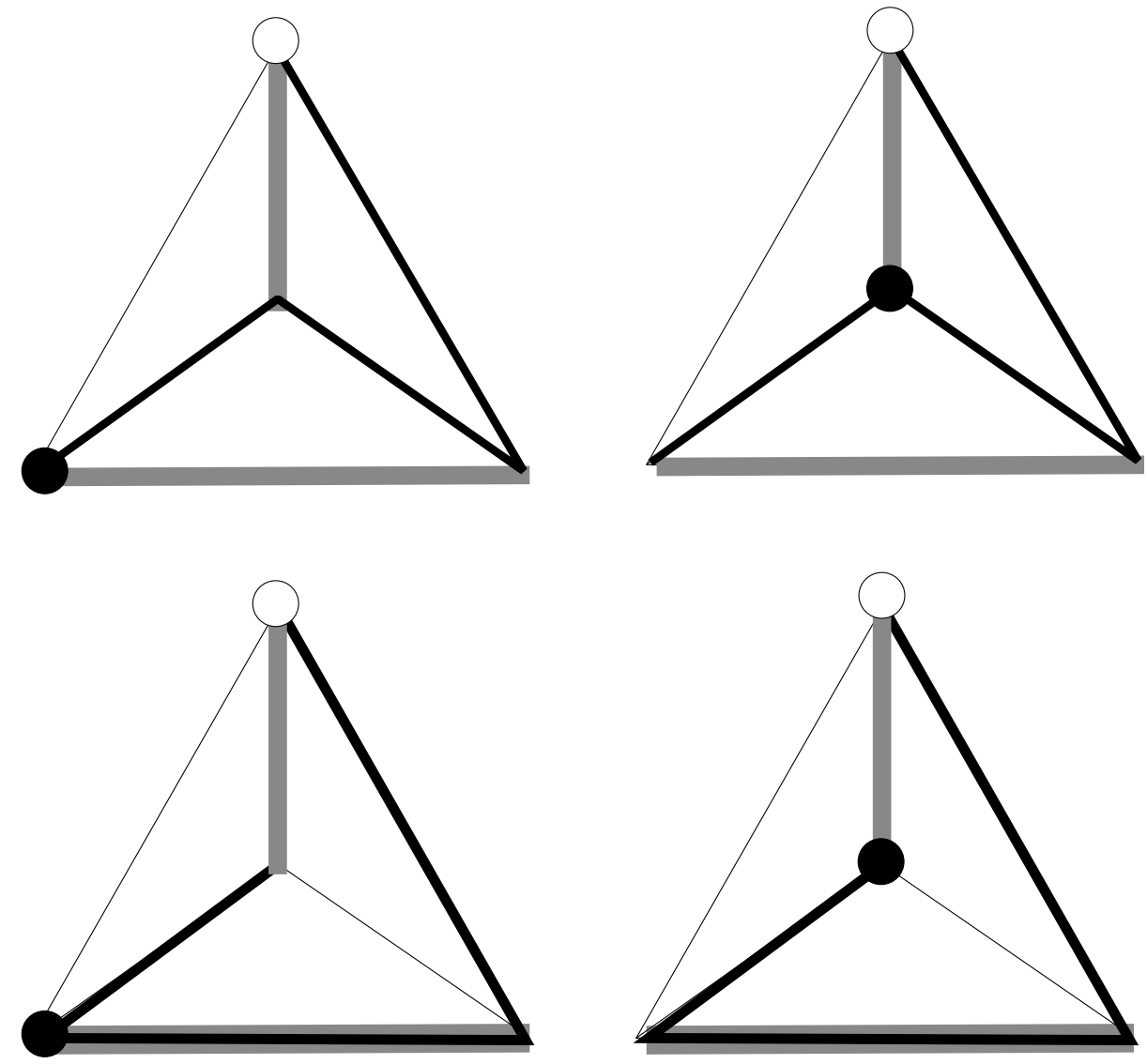

Figure 5.3: Three of the 12 decorations for $X_{\beta}$.

We work with the functions

$$
P=g, \quad Q=6 g-f,
$$

The Positive Dominance algorithm shows that $P \geq 0$ on the above simplices in $473,473,331,331$ steps respectively. The Positive Dominance algorithm shows that $Q \geq 0$ on the above simplices in $467,467,1161,1161$ steps respectively. Our calculations show that $A_{\beta} \leq 0$ and $B_{\beta} \geq 4$.

In fact, $A_{\beta}=0$ and $B_{\beta} \in(4,5)$. I don't know the precise value of $B_{\beta}$ and I'm not sure that the PDA could establish it even if I knew what it was. 


\subsection{Tripods}

Let $\beta$ be a triple of edges incident to the same vertex $v$. Let $X_{\beta}$ be the set of 12 chambers $X_{D}$ such that the black vertex is $v$. That is, the apex of the tripod has the smallest vertex-sum.

We take $\beta=\left\{e_{12}, e_{13}, e_{14}\right\}$. First of all, we anti-certify all the chambers of $X-X_{\beta}$. Now we turn to $X_{\beta}$.

By symmetry, it suffices to consider the first and third simplex listed in the previous section. Both these simplices are contained in the simplex $\boldsymbol{C}_{21}$ from the 12-partition discussed in $\S 2$. We will work with $\boldsymbol{C}_{21}$ because it is just a single simplex. The vertices of $\boldsymbol{C}_{21}$ are

$$
\begin{aligned}
& (4,4,4,4,4,4),(0,8,0,8,0,8),(8,0,0,8,8,0) \\
& (0,0,8,0,8,8),(0,6,6,6,6,0),(6,6,0,0,6,6)
\end{aligned}
$$

We work with the functions

$$
P=4 g-3 f, \quad Q=3 g-f .
$$

The PDA shows that $P \geq 0$ on $\boldsymbol{C}_{21}$ in 967 steps. The PDA shows that $Q \geq 0$ on $\boldsymbol{C}_{21}$ in 779 steps. These calculations show that $A_{\beta} \leq 8$ and $B_{\beta} \geq 18$.

We compute

- $f(8,8,8,8,8,8)=3 \times 2^{12}$.

- $g(8,8,8,8,8,8)=2^{12}$.

- $f(6,6,0,0,6,6)=-2^{7} \times 3^{6}$.

- $g(6,6,0,0,6,6)=-2^{7} \times 3^{5}$.

These calculations show that the two images of $(f, g)$ lie on the two boundary rays of the cones defined by the conditions $P \geq 0$ and $Q \geq 0$. Hence $\left(A_{\beta}, B_{\beta}\right)=(8,18)$.

\subsection{3-paths}

Let $\beta$ be a 3-path. Let $X_{\beta}$ be the set of 8 chambers $X_{D}$ such that the black vertex is an interior vertex of $\beta$, and the outer edges of $D$ are disjoint from $\beta$. In other words, the smallest vertex-sum occurs at an interior vertex of $\beta$ 
and the largest axis-sum occurs on a pair of opposite edges disjoint from $\beta$ (except at the endpoints.)

We take $\beta=\left\{e_{12}, e_{14}, e_{23}\right\}$. First of all, we anti-certify all the chambers of $X-X_{\beta}$. Now we turn to $X_{\beta}$. The chambers of $X_{\beta}$ all lie in $\boldsymbol{C}_{21} \cup \boldsymbol{C}_{13}$. By symmetry, it suffices to consider the chambers in $\boldsymbol{C}_{21}$, the simplex from the previous section. we work with the functions

$$
P=4 g+f, \quad Q=3 g-3 f .
$$

The PDA certifies that $P \geq 0$ in $\boldsymbol{C}_{21}$ in 823 steps. The PDA certifies that $Q \geq 0$ on $\boldsymbol{C}_{21}$ in 1243 steps. These calculations show that $A_{\beta} \leq-6$ and $B_{\beta} \geq 16$.

Remark: I don't know the optimal constants in this case.

\subsection{4-Cycles}

Let $\beta$ be a 4-cycle. Let $X_{\beta}$ be the 16 chambers $X_{D}$ such that the outer two edges of $D$ are disjoint from $\beta$. In other words, the axis-sum of the $\beta$-complement is largest.

We take $\beta=\left\{e_{12}, e_{13}, e_{24}, e_{34}\right\}$. First of all, we anti-certify the chambers of $X-X_{\beta}$. Turning to $X_{\beta}$, we work with the polynomials

$$
P=g, \quad Q=g-f .
$$

In this case, all the chambers of $X_{\beta}$ intersect $X_{24}$ inside the simplex $\boldsymbol{A}_{3}$. By symmetry, it suffices to prove that $P \geq 0$ and $Q \geq 0$ on the simplex $\boldsymbol{C}_{31}$. This simplex has vertices

$$
\begin{aligned}
& (4,4,4,4,4,4),(0,0,8,0,8,8),(8,0,0,8,8,0) \\
& (0,8,0,8,0,8),(0,6,6,6,6,0),(6,0,6,6,0,6)
\end{aligned}
$$

The PDA takes 755 steps to verify that $P \geq 0$ on $\boldsymbol{C}_{31}$ and 1687 steps to verify that $Q \geq 0$ on $C_{31}$. These calculations show that $A_{\beta} \leq 0$ and $B_{\beta} \geq 24$.

Now we observe that

$$
f(4,4,4,4,4,4)=g(4,4,4,4,4,4)=2^{14} .
$$

This forces $B_{\beta}=24$. 
Let $V_{1}, \ldots, V_{6}$ be the vectors listed above and we define

$$
\Theta_{t}=\left(1-t-t^{2}\right) V_{2}+t V_{3}+t^{2} V_{4} .
$$

We compute that

$$
(g+t f)\left(\Theta_{t}\right)=-8388608 t^{7}+O\left(t^{8}\right) .
$$

This shows that $A_{\beta}=0$.

\section{$5.7 \quad 3$-cycles}

We have now completes the proof of Theorem 1.4 and all the auxiliary facts mentioned after that result in the introduction. Now we turn to the proof of Theorem 1.5.

Let $\beta$ be a 3 -cycle in $K_{4}$. Let $X_{\beta}$ be the 36 chambers $X_{D}$ so that the black vertex lies in $\beta$. That is, the vertex with the shortest vertex-sum must be a vertex of $\beta$. We take $\beta=\left\{e_{12}, e_{13}, e_{23}\right\}$. We first anti-certify the chambers of $X-X_{\beta}$.

Now we turn to $X_{\beta}$. By symmetry, it suffices to consider points of $X_{24}$ where the vertex-sum is smallest at vertex 1 . But then we are talking about points in the simplex $\boldsymbol{B}_{1}$. This simplex has vertices

$$
\begin{aligned}
& (0,6,6,6,6,0),(6,0,6,6,0,6),(6,6,0,0,6,6) \\
& (8,0,0,8,8,0),(0,8,0,8,0,8),(0,0,8,0,8,8)
\end{aligned}
$$

We work with the function

$$
P=3 g-f .
$$

The PDA certifies that $P \geq 0$ on $\boldsymbol{B}_{1}$ in 1275 steps.

Let $V_{1}, \ldots, V_{6}$ be the vertices listed above. Define

$$
\Omega_{t}=\left(1-t^{2}\right) V_{1}+t^{2} V_{2}, \quad \Psi_{t}=\left(1-t-t^{2}\right) V_{4}+t V_{1}+t^{2} V_{2} .
$$

We compute that

$$
(3+t) g\left(\Omega_{t}\right)-f\left(\Omega_{t}\right)=-497664 t^{5}+O\left(t^{6}\right) .
$$

Geometrically, this means that when we rotate the line $3 y=x$ about the origin in such a way as to slightly increase its slope, the upper half plane 
bounded by the new line does not contain the image $\boldsymbol{B}_{1}$ under $(f, g)$. We compute that

$$
(3-t) g\left(\Psi_{t}\right)-f\left(\Omega_{t}\right)=663552 t^{6}+O\left(t^{7}\right) .
$$

Geometrically, this means that when we rotate the line $3 y=x$ about the origin in such a way as to slightly decrease its slope, the upper half plane bounded by the new line does not contain the image $\boldsymbol{B}_{1}$ under $(f, g)$. This shows that $g-C f \geq 0$ on $X_{\beta}$ if and only if $C=3$. The corresponding statement in Theorem 1.5 follows from this fact, and from homogeneity.

This completes the proof of Theorem 1.5.

\subsection{The Unfriendly Configurations}

Aside from a 3-cycle, there are two unfriendly configurations, $\alpha$ and $\beta$, chosen so that

$$
K_{4}-\alpha=\left\{e_{12}, e_{13}\right\}, \quad K_{4}-\beta=\left\{e_{12}\right\} .
$$

Some experimental evidence suggests that Corollary 1.6 holds for the set $X_{\alpha}$ of 24 chambers of the form $X_{D}$, where $\alpha \not \subset D$ and the black dot is incident to at least 2 edges of $\alpha$. However, computer plots also show that the image of any chamber $X_{D}$ under the map $(f, g)$ does not lie in a halfspace. (Nonetheless it seems that $f>0$ implies $g>0$.) So, our method simply does not apply here.

Some experimental evidence suggests that Corollary 1.6 holds for a certain set $X_{\beta}$ of 24 chambers whose description in terms of the decorations is rather complicated. 16 of the chambers intersect $X_{24}$ inside $\boldsymbol{A}_{1}$, and the remaining 8 intersect $X_{24}$ inside $\left(\boldsymbol{B}_{3} \cup \boldsymbol{B}_{4}\right)-\boldsymbol{A}_{1}$. The interested reader can download our program and see the set exactly.

Sitting inside $X_{\beta}$ is a smaller set $X_{\beta}^{\prime}$ consisting of the 8 chambers which intersect $X_{24}$ inside $\boldsymbol{C}_{13} \cup \boldsymbol{C}_{14}$. When $X_{D}$ is one of these 8 chambers, the image of $X_{D}$ under $(f, g)$ is contained in a proper cone in $\boldsymbol{R}^{2}$, and the volumeincrease part of Corollary 1.6 holds. We also leave this to the interested reader. 


\section{Appendix: Existence in all Dimensions}

Here I'll give the proof of Theorem 1.2. I learned all the arguments here from Peter Doyle and Igor Rivin. Let $D$ stand for a list $\left\{d_{i j}\right\}$. We will perform operations componentwise, so that $D+t=\left\{d_{i j}+t\right\}$, etc. We define tetrahedral lists in all dimensions just as in the 3 dimensional case. Let $T$ denote the space of tetrahedral lists.

Theorem 5.1 If $D \in T$ then $\sqrt{D} \in T$.

See $[\mathbf{W W}$, Corollary 4.8$]$. In $[\mathbf{W W}]$ this result is attributed to Von Neumann, though Rivin calls it Schoenberg's result.

Theorem 5.2 If $A, B \in T$ then $\sqrt{A^{2}+B^{2}} \in T$. The simplex represented by $\sqrt{A^{2}+B^{2}}$ has larger volume than the simplex represented by $A$.

Proof: See $[\mathbf{R}]$ for a proof. See also [BC, Lemma 1]. Here is a self-contained proof.

Given a quadratic form $Q$ and a linear isomorphism $L$, we have the new quadratic form

$$
L^{*} Q(v, w)=Q\left(L^{-1}(v), L^{-1}(w)\right)
$$

Let $\Delta \subset \boldsymbol{R}^{n}$ denote some copy of the regular simplex. Let $L_{A}$ denote the linear transformation which carries $\Delta$ to a simplex $\Delta_{A}$ whose lengths are realized by $A$. Let $Q_{A}$ be the quadratic form such that $L_{A}^{*}\left(Q_{A}\right)$ is the standard quadratic form - i.e. the dot product. By construction, $Q_{A}$ assigns the length list $A$ to the sides of $\Delta$. Likewise define $Q_{B}$. The sum $Q_{C}=Q_{A}+Q_{B}$ is also positive definite. Given an edge $e$ of $\Delta$. Let $C$ denote the list of lengths that $Q_{C}$ assigns to $\Delta$. We compute

$$
C_{e}=\sqrt{Q_{C}(e, e)}=\sqrt{Q_{A}(e, e)+Q_{B}(e, e)}=\sqrt{A_{e}^{2}+B_{e}^{2}} .
$$

This shows that $Q_{C}$ assigns the corresponding number on the list $\sqrt{A^{2}+B^{2}}$ to the edge $e$. But then let $L_{C}$ be the linear transformation which pushes $L_{C}$ forward to the standard quadratic form. By construction $L_{C}(\Delta)$ is the simplex realizing the list $\sqrt{A^{2}+B^{2}}$.

Note that $L_{C}(e)>L_{A}(e)$ for all $e \in \boldsymbol{R}^{n}$. But then the linear map carrying $\Delta_{A}$ to $\Delta_{C}$ strictly increases all distances, and hence also increases volume.

Let $\phi_{t}$ denote the lengthening flow. That is, $\phi_{t}(D)=D+t$. Let $D_{t}=$ $\phi_{t}(D)$. 


\section{Lemma 5.3}

$$
\frac{d}{d t} \operatorname{vol}\left(D_{t}\right) \geq 0
$$

for all $D \in T$.

Proof: Choose some point $D \in T$. By Theorem 5.1 and scaling, $\sqrt{2 t D} \in T$. By Theorem 5.2. $\sqrt{D^{2}+2 t D} \in T$. But we can write out

$$
\sqrt{D^{2}+2 t D}=D(\sqrt{1+2 t / D})=D+t+\text { higher order terms }
$$

Theorem 5.2 shows that

$$
\operatorname{vol}(D+t+\text { higher order terms })>\operatorname{vol}\left(D_{0}\right)
$$

Taking the limit as $t \rightarrow 0$ we get the result of this lemma.

Lemma 5.3 implies that $D_{t} \in T$ for all $t$, because $\operatorname{vol}\left(D_{t}\right)$ cannot converge to 0 . This takes part of the existence statement in Theorem 1.2.

To see that $\operatorname{vol}\left(D_{t}\right)$ is strictly increasing, we look more carefully at the proof of Theorem 5.2. When all the terms on the list $B$ have size $O(t)$, and $t$ is much smaller than the minimum length on the list $A$, the quadratic form $Q_{C}$ assigns the length $A_{e}+O(t)$ to each unit length vector $e \in \boldsymbol{R}^{n}$. But then the linear map $\Delta_{A}$ to $\Delta_{C}$ increases all unit distances by $O(t)$, and hence increases volume by $O(t)$ as well. This gives

$$
\operatorname{vol}(D+t+\text { higher order terms })>\operatorname{vol}\left(D_{0}\right)+C_{D} t,
$$

for some positive constant $C_{D}$. Taking the limit, we see that $\operatorname{vol}\left(D_{t}\right)$ is strictly increasing, and in fact has positive derivative. This completes the proof of Theorem 1.2. 


\section{References}

[BC] K Bezdek and R. Connelly, Pushing Disks apart - The Kneser-poulsen Conjecture in the plane, Journal fur die riene und angewandte Mathematik (2002) pp 221-236

[L] F. Luo, 3-Dimensional Schlafli Formula and its Generalizations, arXiv:0802.2580

[P] I. Pak, The Cayley-Menger Determinant, UCLA course notes (2006) http://www.math.ucla.edu/ pal/courses/geo/cm.pdf

[Sa] I. Kh. Sabitov, Algebraic methods for solution of polyhedra, Russian Math Surveys 66.3 (2011) pp 445-505

[R] I. Rivin, Some Observations on the Simplex, preprint (2003) arXiv 0308239

[S] R. Schwartz, The Projective Heat Map on Pentagons, research monograph (2014) preprint

[W], S. Wolfram, Mathematica, Wolfram Media and Cambridge University Press (1999)

[WW] J. H. Wells and L. R. Williams, Embeddings and Extensions in Analysis, Springer-Verlag 1975 